\title{
Probiotic-Based Therapy for Active Tuberculosis Infection: The Role of Gut-Lung Axis and Granulocyte Macrophage-Colony Stimulating Factor
}

\author{
Made Indira Dianti Sanjiwani ${ }^{1 *}$, Nyoman Budhi Wirananda Setiawan', Agus Indra Yudhistira Diva Putra ${ }^{1}$, \\ Agus Eka Darwinata ${ }^{2}$ \\ ${ }^{1}$ Faculty of Medicine, Udayana University, Bali, Indonesia. \\ ${ }^{2}$ Department of Microbiology, Faculty of Medicine, Udayana University, Bali, Indonesia.
}

\section{ARTICLE INFO}

Article history:

Received 23 March 2021

Received in revised form 05 May

2021

Accepted 26 May 2021

Available online 31 May 2021

\section{Keywords:}

Gut-lung axis,

Granulocyte macrophage-colony

stimulating factor,

Probiotic,

Tuberculosis.

\begin{abstract}
Tuberculosis is a global health problem with a total of 1.4 million cases in 2015. Over the last decade, several studies have demonstrated the potential role of gut-lung axis in the treatment of tuberculosis. The exact mechanism of the gut-lung axis on tuberculosis is still unknown, however modulation of the gut-lung axis can be performed via probiotic administration. The administered probiotics are capable of inducing an immunomodulating effect which helps in the process of tuberculosis infection. One of the molecules that can be activated with probiotics and plays a role in tuberculosis infection is granulocyte macrophage-colony stimulating factor (GM-CSF). GM-CSF can control intracellular production of M. tuberculosis, inflammation in granulomas, and lung tissue reparation. This article aimed to explore the role of the gut-lung axis, GM-CSF, and the potential of probiotic-based therapy on active tuberculosis infection. It was found that probiotics mediate the immune response via the activation of several inflammatory cytokines and interleukins related to lung infection, but not directly with the tuberculosis pathogen. Thus, probiotic-based therapy has the potential to increase immunity during active tuberculosis infection. Further studies to explore the other mechanisms of the gut-lung axis against tuberculosis through probiotic administration need to be performed.
\end{abstract}

\section{INTRODUCTION}

Tuberculosis is an infectious disease caused by Mycobacterium tuberculosis. Based on World Health Organization (WHO) data, in 2015 tuberculosis recorded the highest number of cases, namely 1.4 million cases. ${ }^{1}$ Meanwhile in Indonesia, the number of cases has increased every year and has reached 420,994 people in 2017. Even the mortality rate for tuberculosis has increased to 1.3 million people in $2017 .^{2}$

Management of tuberculosis is focusing on the anti-tuberculosis drug regimen which consists of four types of drugs that are taken for six months. Prolonged consumption of the anti-tuberculosis drug regimen may cause a decrease in patient adherence which can also affect the success of therapy and lead to M. tuberculosis resistance to the anti-tuberculosis drug. ${ }^{3}$ The incidence of resistance to the first-line anti-tuberculosis drug, known as multiple drug resistance tuberculosis (MDR$\mathrm{TB}$ ), and resistance to the first and second line, known as extensively drug resistance tuberculosis (XDR-TB), shows an increase over time and is a global health problem today. ${ }^{4}$ In addition, taking this anti-tuberculosis drug regimen over a long period often results in decreased patient adherence which can also contribute to resistance. ${ }^{3}$ This indicates the urgency to find innovative therapeutic methods that can solve the issue of resistance to the anti-tuberculosis drug.

\footnotetext{
*Correspondence: indiradianti17@gmail.com
} 
In the last few years, therapeutic innovation methods have been proposed in the management of tuberculosis, one of the potential mechanisms being discovered is the gut-lung axis. The gut-lung axis is a mechanism that shows the interplay between two distant organs, namely the intestines and lungs. This is related to abnormalities in the balance of the intestinal microbiota which can affect the situation of the lungs and vice versa. ${ }^{5}$ Previous studies have shown that regulation of colonization of the gut microbiota has an effect on the maturity of the respiratory tract. ${ }^{6}$

Changes in the balance of the gut microbiota composition are known to occur in cases of tuberculosis, but it is not clear whether this change is due to tuberculosis infection, tuberculosis therapy, or is a predisposition that can precipitate pathogen development. Several studies have demonstrated the role of gut microbiota in the management of tuberculosis. The mechanisms that are induced include a reduction in inflammation and strengthening of immunity. ${ }^{7}$ However, the comprehensive mechanism related to the induction of the gut microbiota against tuberculosis is not fully understood. One potential mechanism is through the activation of granulocyte-colony stimulating factor (GM-CSF). GM-CSF is activated in the body as an immune response, a myelopoiesis regulator, and is known to play a significant role in the inflammatory process. Previous studies have shown that GM-CSF can reduce intracellular $M$. tuberculosis production and control inflammation in granulomas. ${ }^{8}$

Seeing the potential of the gut microbiota against GM-CSF activation opens an opportunity to find tuberculosis therapy with gut-lung axis modulation. One of the ways to modulate the gut-lung axis is through the administration of probiotics. Probiotics are good bacteria where previous studies have found probiotics are not only able to suppress inflammation in tuberculosis but also have a protective effect against anti-tuberculosis drug-induced hepatotoxicity. ${ }^{9,} 10$ Therefore, this literature review will discuss the potential of probiotic-based therapy through GM-CSF activation in the management of active tuberculosis.

\section{Gut-lung axis and its role in tuberculosis pathogenesis}

Trillions of microbiota colonies exist in the human body physiologically, namely what is called normal flora. Normal flora has a role in body homeostasis and is present in several parts of the body including the intestines and lungs. Changes in the composition of the microbiota or dysbiosis, in both lungs and intestines, were found to be associated with dysregulation of the immune response and disease progression in the lungs. ${ }^{11}$ Previous studies shown that improving the composition of the gut microbiota can reduce the severity of the respiratory disease. This suggests the essential role of the gut-lung axis in respiratory disease. The gut-lung axis can provide a feedback mechanism between the intestines and lungs, either through translocation of the microbiota or through the release of immunomodulatory metabolites such as short-chain fatty acids (SCFA). Mesenteric blood and lymphatic circulation play an important role in the gutlung axis because it becomes a translocation pathway for metabolites, immune signals, and microbiota from the intestine to the lungs and vice versa. ${ }^{12}$

The gut-lung axis also plays a role in the pathogenesis of tuberculosis. Previous studies reported that tuberculosis patients who were given antituberculosis drugs experienced dysbiosis or a substantial change in the composition of the gut microbiota. The gut microbiota of a tuberculosis patient treated with isoniazid, rifampin, and pyrazinamide (HRZ) were predominantly erysipelatoclostridium and eggerthia. ${ }^{13}$ In addition, a study by Mendonca, et al., showed that antituberculosis drugs administration is capable of causing intestinal dysbiosis which then affects the response of alveolar macrophages to tuberculosis infection. ${ }^{14}$ This indicates the role of the gut-lung axis in the pathogenesis of tuberculosis, especially in modulating the immune response.

The mechanism of immunity control via the gutlung axis can be seen in Figure 1. The intestinal microbiota induces signaling changes and induces the migration of dendritic cells (DC) to the lymphatic tract. ${ }^{15} \mathrm{DC}$ then plays a role in the process of activating various $\mathrm{T}$ cells in the mesenteric lymph nodes (MLN) and inducing the production of cytokines such as IL-10 TGF- $\beta$, INF $\gamma$, and IL- $6 .{ }^{16}$ Activated T cells then occupy their receptors and when there is an infection reaction in the respiratory tract, cells from the MLN and gutassociated lymphoid tissue (GALT) will migrate to the lung mucosa and provides a protective and antiinflammatory effect. The microbiota product in the form 


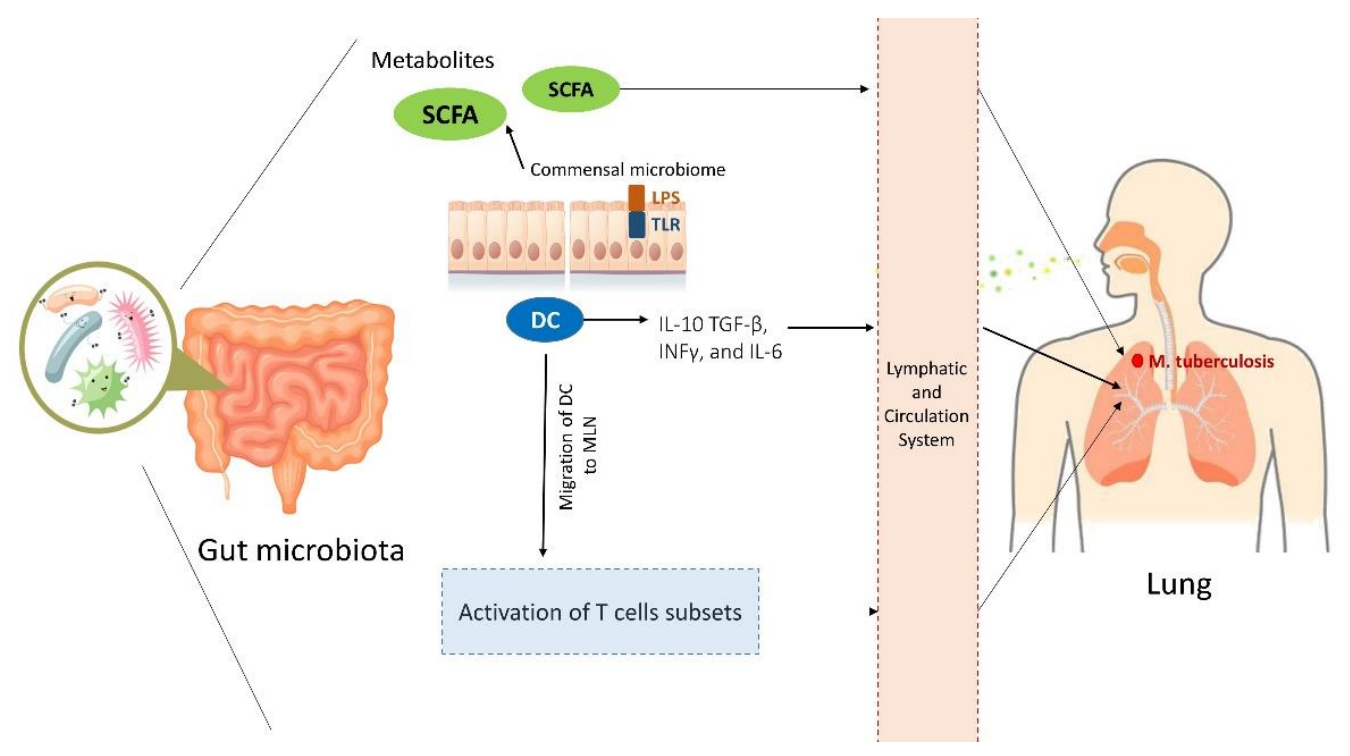

Figure 1. Gut-lung axis mechanism in immune response against Mycobacterium tuberculosis

of lipopolysaccharide (LPS) can bind to TLR on intestinal epithelial cells and macrophages which are then also able to induce cytokine production and chemokine. Apart from its products, the microbiota metabolite in the form of SCFA was also found to play a role in the gut-lung axis because it may translocates to the lungs. ${ }^{17}$

\section{Granulocyte macrophage-colony stimulating factor (GM-CSF)}

GM-CSF is one of the cytokine family of colonystimulating factors (CSFs) which is a regulator of myelopoiesis. Apart from its function in the development of alveolar macrophage maturation and nonlymphoid dendritic cells, GM-CSF also has a dominant function in the inflammatory process. When inflammation occurs, the myeloid responds by activating T cells that are known to produce GM-CSF. ${ }^{18}$

GM-CSF production is not only limited by $\mathrm{T}$ cells, but also by several other cells such as epithelial cells, leukocytes, and fibroblasts. GM-CSF that has been produced will bind to the GM-CSF Receptor (GMCSFR) then induce several cascades that play an important role in the production of immune and inflammatory genes, namely JAK-STAT, mitogenactivated protein kinase (MAPK), NF-KB, and phosphatidylinositol 3-kinase (PI3K). ${ }^{19}$ Inflammation is an immune reaction to immunogenic substances originating from outside (infection) and in the body (autoimmune). ${ }^{20}$ Inflammation has a protective function but is also pathological in most situations, one of which is tuberculosis.

Tuberculosis is caused by $M$. tuberculosis infection, which is an intracellular pathogen. Generally, tuberculosis does not cause clinical manifestations at first, occurs gradually, and may become latent. The protective effect of inflammation plays an important role in latent tuberculosis, where the absence of myeloid or $\mathrm{T}$ cells puts the body in an immunodepletion state and can lead to active tuberculosis. ${ }^{21}$ In a pathological context, inflammation is able to increase the immunopathological survival of tuberculosis. GM-CSF in tuberculosis has a protective role, where GM-CSF is expressed in tuberculous granulomas and secreted by macrophages and lung epithelial cells. ${ }^{22}$

In the absence of GM-CSF, the rat hematopoiesis was reported to be undisturbed but dysregulated in the surfactant recycle process in alveolar macrophages, and showed signs of inflammation in the lungs that resembled pulmonary alveolar proteinosis (PAP). ${ }^{23}$ Another study showed that in the absence of GM-CSF, mice were unable to restrict the growth of $M$. tuberculosis, had problems with lymphocyte recruitment, and were unable to form normal granulomas. ${ }^{24}$ Previous studies have also tested the effectiveness of giving recombinant-human-GM-CSF (Rhu-GM-CSF) recombinant in patients with active tuberculosis and had earlier negative tuberculosis results in the intervention group. ${ }^{25}$ This indicates the significance of the presence of GM-CSF when tuberculosis infection is active. 
Gut-lung axis mechanism in protection against tuberculois through GM-CSF activation

The gut microbiota through the gut-lung axis is known to have a protective effect against pulmonary tuberculosis. The comprehensive mechanism regarding the gut-lung axis against tuberculosis is still being investigated. Until now, the protective mechanism of the gut-lung axis against tuberculosis was known through induction of immunity (Figure 1). Several studies suggest that one of the mechanisms induced by the gut microbiota is the activation of GM-CSF. ${ }^{8,24}$

Commensal gut microbiota plays a role in mediating innate immunity against pathogens. When tuberculosis infection occurs, the gut microbiota will stimulate different immune cells, such as mucosaassociated invariant $\mathrm{T}$ (MAIT) cells, group 3 innate lymphoid cells (ILC3), and natural killer (NK) cells to induce protective immunity in the lungs. ${ }^{26} \mathrm{NK}$ and MAIT cells when stimulated will increase the synthesis of proinflammatory cytokines, namely interleukin-17A (IL-17A), while ILC3 will respond by increasing the production of interleukin-22 (IL-22). These two cytokines play a role in lung protection against tuberculosis through different but the same target, namely in inducing alveolar macrophages to phagocytose pathogens. IL-22 will affect pulmonary epithelial cells and alveolar macrophages infected with M. tuberculosis. IL-22 will bind to its receptor, namely IL-22R1 on lung epithelial cells and stimulate lung tissue repair \& healing, decrease infection susceptibility, and proliferation of alveolar epithelial cells. Meanwhile, in alveolar macrophages infected with M. tuberculosis, IL-22 has the effect of increasing the production of antimicrobial peptides and inhibiting mycobacterium growth. IL-17A, on the other hand, exerts a protective effect through activation and increased production of GM-CSF. ${ }^{27}$

Increased production of GM-CSF by the gut microbiota is mediated by the role of IL-17A. Increased GM-CSF stimulates pathogen elimination through several mechanisms, such as phagocytosis, decreased reactive oxygen species (ROS), and extracellular signalregulated kinase (ERK) signaling. This ERK signaling will have a control effect on granuloma inflammation as well as control the growth of M. tuberculosis. Macrophages will later secrete IL-1 $\beta$ which is antimicrobial and can act as a cofactor in the maturation and action of Th17 cells. Th17 cells are also recognized as a major producer of IL-17A, which in turn can reactivate GM-CSF (Figure 2). ${ }^{28,29}$

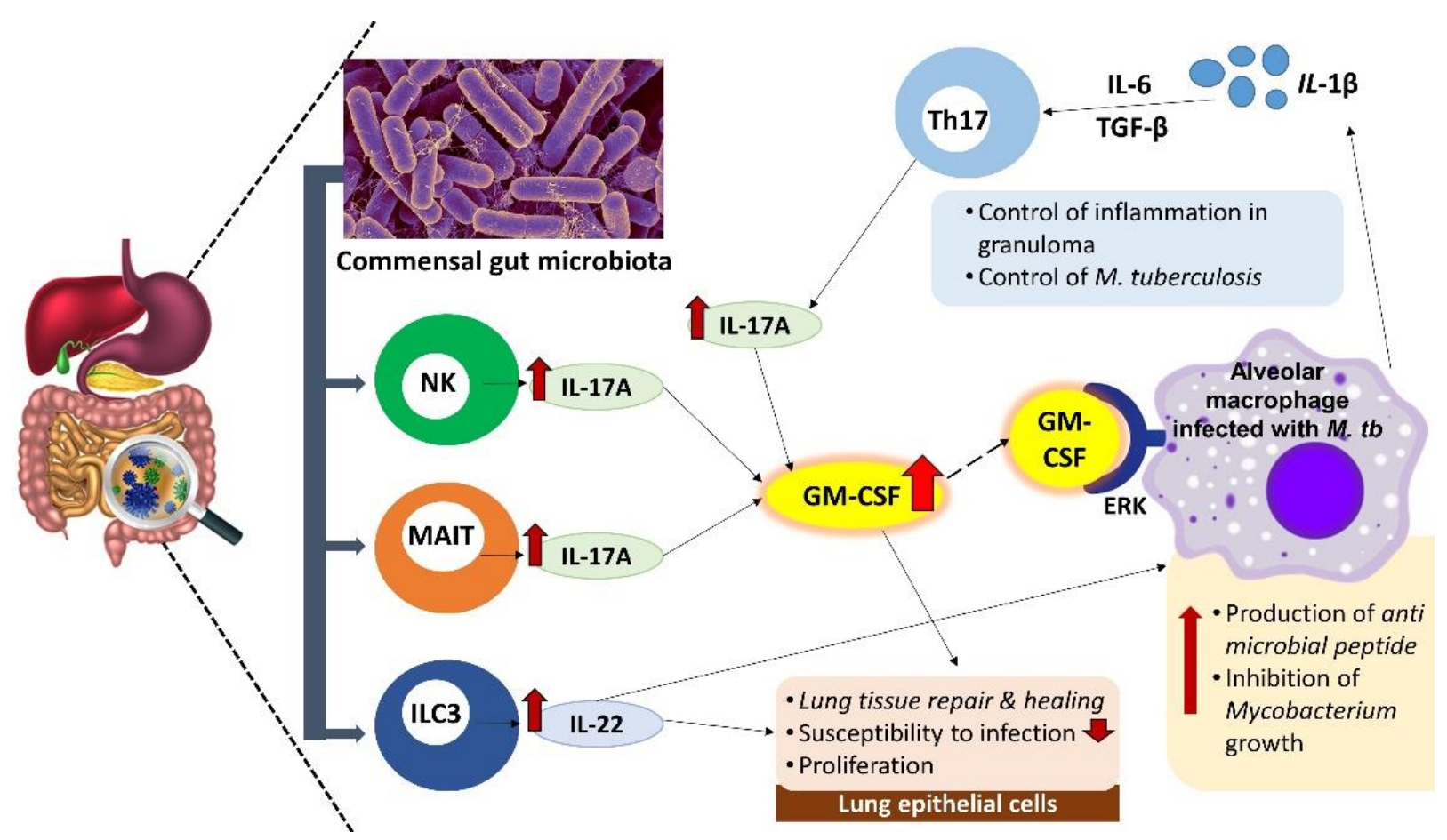

Figure 2. The mechanism of gut microbiota against tuberculosis infection through GM-CSF activation ${ }^{26-29}$ 
Probiotic in modulating the gut-lung axis as a potential therapy for active tuberculosis infection

The modulation of the gut-lung axis can be performed by several methods. One of the simplest and widely studied methods is the administration of probiotics. Probiotics play a role in the management of tuberculosis through their mechanism in the activation of GM-CSF. A previous study by Rothchild, et al. demonstrated that GM-CSF helps increase antimicrobial activity against pathogens. ${ }^{8}$ Several studies regarding the administration of probiotics and its effects on cytokine and interleukin that is related to tuberculosis and lung infection have been summarized in Table 1.

Activation of GM-CSF is one of the steps induced by the intestinal microbiota in a homeostatic state. Previous studies in a group of mice inoculated with gram-positive Streptococcus pneumoniae and a group of gram-negative Klebsiella pneumoniae mice showed that antibiotic administration led to a decrease in GM-CSF, chemokine (CXC motif) ligand 2 (CXCL2), and CXCL1, which are important signaling molecules in the innate immune response against pulmonary infection. The study also showed an increased increase in bacterial colonyforming units (CFU) in the antibiotic-treated group of mice compared to the control group (without giving the antibiotic). ${ }^{30}$ This indicates that it induces disruption of response and increases in pathogens in an acute period.

Disruption of the composition of the microbiota due to antibiotic administration occurs in the administration of the anti-tuberculosis drug and can cause a decrease in the immune response. Several previous studies have tested the administration of probiotics for lung disease in both the pre-clinical and clinical stages. A study by Suprapti, et al. examined the administration of multistrain probiotics and B vitamins in intensive phase tuberculosis patients where there were more significant increases in IFN- $\gamma$ and IL-12 in the supplemented group. ${ }^{31}$ This indicates a strengthening of the immune system where the increase in IFN- $\gamma$ and IL12 plays a role in helping the eradication of $M$. tuberculosis.

Administration of probiotics in the form of a group of nucleotide-binding oligomerization domaincontaining protein 2 (NOD2) stimulating bacteria plays a role in immunity by reducing TNF- $\alpha$ and IL- $1 \beta$ in bronchoalveolar lavage fluid (BALF). ${ }^{32}$ NOD2 is known to modulate the immune response through activation of GM-CSF. An in-vivo study examined intragastric administration of the probiotic Lactobacillus paracase $i$ CNCM I-1518 and resulted in increased pulmonary IL$10 .^{33}$ Similar studies with the administration of eight different Lactobacillus strains showed a high survival rate of mice against lung pathogens, where protection was highest by Lactobacillus fermentum-1. This is because Lactobacillus triggers an increase in $\operatorname{IgA}$ as well as a decrease in IL- 6 and TNF- $\alpha$ in the lungs. ${ }^{34}$

Another study exploring intragastric administration of Lactobacillus rhamnosus GG showed a decreased IL- 6 , decreased TNF- $\alpha$, increased serum and pulmonary IL-10. It also increases regulatory $\mathrm{T}$ cells and increases neutrophil infiltration in the lungs by decreasing the expression of TLR2 and MyD88 genes. This will then decrease the immune response if it is excessive, thus lung injury can be minimized. ${ }^{35}, 36$ Administration of live Bifidobacterium longum 51A can decrease TNF- $\alpha$ and IL- 6 in BALF, increase IL-10 in the lungs, and increase the phagocytosis ability of alveolar macrophages via increased ROS. $^{37}$ In addition, a Randomized Clinical Trial (RCT) study in testing the effect of probiotics on immunity showed significant results in neutrophil resting burst $(2.6-3.2 \%, \mathrm{p}=0.0134)$ and serum neopterin levels (7.7-8.4 nmol / L, p = $0.001) .{ }^{10}$ This shows the potential of probiotics in modulating immunity via the gut-lung axis to fight pathogens in the lung.

Probiotics also induce hepatoprotective effects and a positive gastrointestinal effect. The antituberculosis drug is known to induce toxic effects on the liver and the administration of probiotics can provide a protective effect against this phenomenon. ${ }^{10}$ Previous studies have also shown that the probiotic Lactobacillus casei can prevent the effects of nausea and decrease appetite in patients who are given OAT. ${ }^{38}$ In addition, all of the studies of probiotic administration included in this review article did not report any side effects or adverse events caused by the intervention. This indicates that the administration of probiotics is relatively safe in cases of lung infection. 
Table 1. Several studies of probiotic administration and its effects on lung infection related-cytokines and interleukins

\begin{tabular}{|c|c|c|c|}
\hline Author (year) & Intervention & Effects & Ref \\
\hline Suprapti, et al. (2018) & Multistrain probiotic + vitamin B & Increased IFN- $\gamma$ and IL-12 & 31 \\
\hline Belkacem, et al. (2017) & $\begin{array}{l}\text { Probiotic Lactobacillus paracasei } \mathrm{CNCM} \\
\mathrm{I}-1518\end{array}$ & Increased IL-10 in lung tissues & 32 \\
\hline Robak, et al. (2018) & Probiotic of NOD2-stimulating bacteria & Decreased TNF- $\alpha$ and IL- $1 \beta$ in BALF & 33 \\
\hline $\begin{array}{l}\text { Youn, et al. } \\
\text { (2012) }\end{array}$ & Probiotic Lactobacillus fermentum-1 & $\begin{array}{l}\text { Increased IgA } \\
\text { Decreased IL- } 6 \text { and TNF- } \alpha\end{array}$ & 34 \\
\hline Khailova, et al. (2013) & Probiotic Lactobacillus rhamnosus GG & Decreased IL-6 and TNF- $\alpha$ & 35 \\
\hline Martens, et al. (2018) & Probiotic Lactobacillus rhamnosus GG & $\begin{array}{l}\text { Increased IL-10 in serum and lung tissues } \\
\text { Increased T cell regulator and neutrophil } \\
\text { infiltration in lung, and decreased expression of } \\
\text { TLR2 gene and Myd } 88\end{array}$ & 36 \\
\hline Vieira, et al. (2016) & Probiotic Bifidobacterium longum 51A & $\begin{array}{l}\text { Decreased TNF- } \alpha \text { and IL- } 6 \text { in BALF } \\
\text { Incerased IL-10 in lung tissues, macrophage } \\
\text { paghocytosis ability }\end{array}$ & 37 \\
\hline
\end{tabular}

\section{SUMMARY}

Probiotic-based therapy has a role on the immune system during active tuberculosis infection through its various mechanisms, namely controlling the growth of M. tuberculosis, controlling granuloma inflammation, and lung tissue repair. In addition to the effects on immunity against tuberculosis, probiotics are also able to induce hepatoprotective effects and treat gastrointestinal side effects due to anti-tuberculosis drugs. The effectiveness and direct efficacy of probiotic-based therapy in tuberculosis cases have yet to be found, but its potential as an adjuvant in the treatment of tuberculosis still needs to be investigated.

\section{REFERENCES}

1. WHO. Global Tuberculosis Report 2018. Global Tuberculosis Report 2017. 2017.

2. Depkes RI. Infodatin Tuberculosis. Kementerian Kesehatan RI. 2018;1.

3. AlSahafi AJ, Shah HBU, AlSayali MM, Mandoura N, Assiri M, Almohammadi EL, et al. High NonCompliance Rate with Anti-Tuberculosis Treatment: A Need to Shift Facility-Based Directly Observed Therapy Short Course (DOTS) to Community Mobile Outreach Team Supervision in Saudi Arabia. BMC Public Health. 2019;19(1):1168.

4. Simmons JD, Stein CM, Seshadri C, Campo M, Alter G, Fortune S, et al. Immunological Mechanisms of Human Resistance to Persistent Mycobacterium tuberculosis Infection. Nature Reviews Immunology. 2018;18(9):575-89.

5. He Y, Wen Q, Yao F, Xu D, Huang Y, Wang J. Gut-Lung Axis: The Microbial Contributions and Clinical Implications. Critical Reviews in Microbiology. 2017;43(1):81-95.

6. Li W, Zhu Y, Liao Q, Wang Z, Wan C. Characterization of Gut Microbiota in Children with
Pulmonary Tuberculosis. BMC Pediatrics. 2019;19(1):445.

7. Dumas A, Corral D, Colom A, Levillain F, Peixoto A, Hudrisier D, et al. The Host Microbiota Contributes to Early Protection against Lung Colonization by Mycobacterium tuberculosis. Frontiers in Immunology. 2018;9:2656.

8. Rothchild AC, Stowell B, Goyal G, Nunes-Alves C, Yang Q, Papavinasasundaram K, et al. Role of Granulocyte-Macrophage Colony-Stimulating Factor Production by $\mathrm{T}$ Cells during Mycobacterium tuberculosis Infection. MBio. 2017;8(5).

9. Zolnikova OY, Ivaschkin K, Bueverova E, Ivaschkin V. Intestinal Microbiota, Nutrients and Probiotics Viewed from the «Gut-Lung» Axis. Voprosy Pitaniia. 2019;88(3):13-22.

10. Horvath A, Leber B, Schmerboeck B, Tawdrous M, Zettel G, Hartl A, et al. Randomised Clinical Trial: The Effects of a Multispecies Probiotic vs. Placebo on Innate Immune Function, Bacterial Translocation and Gut Permeability in Patients with Cirrhosis. Alimentary Pharmacology \& Therapeutics. 2016;44(9):926-35.

11. McAleer JP, Kolls JK. Contributions of the Intestinal Microbiome in Lung Immunity. European Journal of Immunology. 2018;48(1):39-49.

12. Ma Y, Yang X, Chatterjee V, Wu M, Yuan SY. The Gut-Lung Axis in Systemic Inflammation: Role of Mesenteric Lymph as Conduit. American Journal of Respiratory Cell and Molecular Biology. 2020(ja).

13. Hu Y, Yang Q, Liu B, Dong J, Sun L, Zhu Y, et al. Gut Microbiota Associated with Pulmonary Tuberculosis and Dysbiosis Caused by AntiTuberculosis Drugs. Journal of Infection. 2019;78(4):317-22.

14. Khan N, Mendonca L, Dhariwal A, Fontes G, Menzies D, Xia J, et al. Intestinal Dysbiosis Compromises Alveolar Macrophage Immunity to Mycobacterium tuberculosis. Mucosal immunology. 2019;12(3):772-83.

15. Negi S, Pahari S, Bashir H, Agrewala JN. Gut Microbiota Regulates Mincle Mediated Activation of Lung Dendritic Cells to Protect against 
Mycobacterium tuberculosis. Frontiers in Immunology. 2019;10:1142.

16. Geva-Zatorsky N, Sefik E, Kua L, Pasman L, Tan TG, Ortiz-Lopez A, et al. Mining the Human Gut Microbiota for Immunomodulatory Organisms. Cell. 2017;168(5):928-43. e11.

17. Samuelson DR, Welsh DA, Shellito JE. Regulation of Lung Immunity and Host Defense by the Intestinal Microbiota. Frontiers in Microbiology. 2015;6:1085.

18. Hamilton JA. GM-CSF-Dependent Inflammatory Pathways. Frontiers in Immunology. 2019;10:2055.

19. Robinson RT. T Cell Production of GM-CSF Protects the Host during Experimental Tuberculosis. MBio. 2017;8(6).

20. Piergallini TJ, Turner J. Tuberculosis in the Elderly: Why Inflammation Matters. Experimental Gerontology. 2018;105:32-9.

21. Scriba TJ, Penn-Nicholson A, Shankar S, Hraha T, Thompson EG, Sterling D, et al. Sequential Inflammatory Processes Define Human Progression from M. tuberculosis Infection to Tuberculosis Disease. PLoS pathogens. 2017;13(11):e1006687.

22. Benmerzoug S, Marinho FV, Rose S, Mackowiak C, Gosset D, Sedda D, et al. GM-CSF Targeted Immunomodulation Affects Host Response to $\mathrm{M}$. tuberculosis Infection. Scientific Reports. 2018;8(1):1-15.

23. Tazawa R, Ueda T, Abe M, Tatsumi K, Eda R, Kondoh S, et al. Inhaled GM-CSF for Pulmonary Alveolar Proteinosis. New England Journal of Medicine. 2019;381(10):923-32.

24. Bryson BD, Rosebrock TR, Tafesse FG, Itoh CY, Nibasumba A, Babunovic GH, et al. Heterogeneous GM-CSF Signaling in Macrophages is Associated with Control of Mycobacterium tuberculosis. Nature Communications. 2019;10(1):1-11.

25. Kaufmann SH, Lange C, Rao M, Balaji KN, Lotze $\mathrm{M}$, Schito $\mathrm{M}$, et al. Progress in Tuberculosis Vaccine Development and Host-Directed Therapies-A State of the Art Review. The Lancet Respiratory Medicine. 2014;2(4):301-20.

26. Gupta N, Kumar R, Agrawal B. New Players in Immunity to Tuberculosis: The Host Microbiome, Lung Epithelium, and Innate Immune Cells. Frontiers in Immunology. 2018;9:709.

27. Mishra A, Singh VK, Actor JK, Hunter RL, Jagannath C, Subbian S, et al. GM-CSF Dependent Differential Control of Mycobacterium tuberculosis Infection in Human and Mouse Macrophages: Is Macrophage Source of GM-CSF Critical to Tuberculosis Immunity? Frontiers in Immunology. 2020;11:1599.

28. Cheng H, Guan X, Chen D, Ma W. The Th17/Treg Cell Balance: A Gut Microbiota-Modulated Story. Microorganisms. 2019;7(12):583.
29. Khan R, Petersen FC, Shekhar S. Commensal Bacteria: An Emerging Player in Defense against Respiratory Pathogens. Frontiers in Immunology. 2019;10:1203.

30. Brown RL, Sequeira RP, Clarke TB. The Microbiota Protects against Respiratory Infection via GM-CSF Signaling. Nature Communications. 2017;8(1):1-11.

31. Suprapti B, Suharjono S, Raising R, Yulistiani Y, Izzah Z, Nilamsari WP, et al. Effects of Probiotics and Vitamin B Supplementation on IFN- $\gamma$ and IL-12 Levels During Intensive Phase Treatment of Tuberculosis. Indonesian Journal of Pharmacy. 2018;29(2):80.

32. Robak OH, Heimesaat MM, Kruglov AA, Prepens $\mathrm{S}$, Ninnemann $\mathrm{J}$, Gutbier $\mathrm{B}$, et al. Antibiotic Treatment-Induced Secondary IgA Deficiency Enhances Susceptibility to Pseudomonas aeruginosa pneumonia. The Journal of Clinical Investigation. 2018;128(8):3535-45.

33. Belkacem N, Serafini N, Wheeler R, Derrien M, Boucinha L, Couesnon A, et al. Lactobacillus paracasei Feeding Improves Immune Control of Influenza Infection in Mice. PloS one. 2017;12(9):e0184976.

34. Youn H-N, Lee D-H, Lee Y-N, Park J-K, Yuk S-S, Yang S-Y, et al. Intranasal Administration of Live Lactobacillus Species Facilitates Protection against Influenza Virus Infection in Mice. Antiviral Research. 2012;93(1):138-43.

35. Khailova L, Baird CH, Rush AA, McNamee EN, Wischmeyer PE. Lactobacillus rhamnosus GG Improves Outcome in Experimental Pseudomonas aeruginosa pneumonia: Potential Role of Regulatory T cells. Shock (Augusta, Ga). 2013;40(6):496.

36. Martens K, Pugin B, De Boeck I, Spacova I, Steelant B, Seys S, et al. Probiotics for the Airways: Potential to Improve Epithelial and Immune Homeostasis. Allergy. 2018;73(10):1954-63.

37. Vieira AT, Rocha VM, Tavares L, Garcia CC, Teixeira MM, Oliveira SC, et al. Control of Klebsiella pneumoniae Pulmonary Infection and Immunomodulation by Oral Treatment with the Commensal Probiotic Bifidobacterium longum 51A. Microbes and Infection. 2016;18(3):180-9.

38. Lin S, Zhao S, Liu J, Zhang J, Zhang C, Hao H, et al. Efficacy of Proprietary Lactobacillus casei for Anti-Tuberculosis Associated Gastrointestinal Adverse Reactions in Adult Patients: A Randomized, Open-Label, Dose-Response Trial. Food \& Function. 2020;11(1):370-7. 